\title{
NEIGHBOURHOODS OF A CERTAIN SUBCLASS OF UNIFORMLY STARLIKE FUNCTIONS
}

\author{
T. SRINIVAS, P. THIRUPATHI REDDY AND B. MADHAVI
}

\begin{abstract}
In this paper, we introduced new subclasses $U C V_{S}(\alpha, \beta)$ and $S P_{S}(\alpha, \beta)$ which are sub classes of $U C V(\alpha, \beta)$ and $S P(\alpha, \beta)$ and studied the $T-\delta$ neighbourhoods of functions in these classes. The results obtained in this paper generalizes the recent results of Parvatham and Premabai [5], Ram Reddy and Thirupathi Reddy [6, 7], Padmanabhan [4], and Ronning [8].
\end{abstract}

\section{Introduction}

Let $\boldsymbol{A}$ denote the class of functions of the form

$$
f(z)=z+\sum_{n=2}^{\infty} a_{n} z^{n}
$$

which are analytic in the unit disk $E=\{z:|z|<1\}$. Further, let $\boldsymbol{S}$ be the subclass of $\boldsymbol{A}$ consisting of those functions that are univalent in $E$. Let $C V$ and $S T$ denote the subclasses of $S$ consisting of convex and starlike functions respectively.

If $f(z)=z+\sum_{n=2}^{\infty} a_{n} z^{n}$ and $g(z)=z+\sum_{n=2}^{\infty} b_{n} z^{n}$ then the convolution or Hadamard product of $f(z)$ and $g(z)$ denoted by $f * g$ is defined by

$$
(f * g)(z)=z+\sum_{n=2}^{\infty} a_{n} b_{n} z^{n}
$$

Clearly

$$
f(z) * \frac{z}{(1-z)^{2}}=z f^{\prime}(z) \quad \text { and } f(z) * \frac{z}{\left(1-z^{2}\right)}=\left[\frac{f(z)-f(-z)}{2}\right] .
$$

Goodman [1,2] defined the following subclasses of $C V$ and $S T$.

Definition A. A function $f$ is uniformly convex (Starlike) in $E$ if $f$ is in $C V(S T)$ and has the property that for every circular arc $\gamma$ contained in $E$ with centre $\xi$ also in $E$, the arc $f(\gamma)$ is convex (Starlike w.r.t $f(\xi)$ ).

Corresponding author: P. Thirupathi Red dy.

Key words and phrases. Neighbourhood, subordination, hadamard product. 
Goodman $[1,2]$ then gave the following two variable analytic characterizations of these classes, denoted by $U C V$ and $U S T$.

Theorem A. A function $f$ of the form (1) is in UCV if and only if

$$
\operatorname{Re}\left\{1+(z-\xi) \frac{f^{\prime \prime}(z)}{f^{\prime}(z)}\right\} \geq 0,(z, \xi) \in E \times E .
$$

and is in UST if any only if

$$
\operatorname{Re}\left\{\frac{f(z)-f(\xi)}{(z-\xi) f^{\prime}(z)}\right\} \geq 0,(z, \xi) \in E \times E .
$$

The classical Alexander result that $f \in C V$ if and only if $z f^{\prime} \in S T$ does not hold between the classes $U C V$ and $U S T$. Ronning [9] defined a subclass of starlike functions $S_{p}$ with the property that a function $f \in U C V$ if and only if $z f^{\prime} \in S_{p}$.

Definition B. Let $S_{p}=\left\{F \in S T \mid F(z)=z f^{\prime}(z), f \in U C V\right\}$.

Ma and Minda [3] and Ronning [9] independently found a more applicable one variable characterization for $U C V$.

Theorem B. A function $f$ is in UCV if and only if

$$
\operatorname{Re}\left\{1+\frac{z f^{\prime \prime}(z)}{f^{\prime}(z)}\right\} \geq\left|\frac{z f^{\prime \prime}(z)}{f^{\prime}(z)}\right|, \quad z \in E .
$$

Ronning [9] proved a one variable characterization for $S_{p}$ as follows:

Theorem C. A function $f$ is in $S_{p}$ if and only if

$$
\left|\frac{z f^{\prime}(z)}{f(z)}-1\right| \leq \operatorname{Re}\left\{\frac{z f^{\prime}(z)}{f(z)}\right\}, z \in E .
$$

A function $f \in A$ is uniformly convex of order $\alpha$ for $-1 \leq \alpha<1$ if and only if $1+\frac{z f^{\prime \prime}(z)}{f^{\prime}(z)}$ lies in the parabolic region

$$
\operatorname{Re}\{\omega-\alpha\}>|\omega-1|
$$

In otherwords, the function $f$ is uniformly convex of order $\alpha$ if

$$
1+\frac{z f^{\prime \prime}(z)}{f^{\prime}(z)} \prec 1+\frac{2(1-\alpha)}{\pi^{2}}\left[\log \left(\frac{1+\sqrt{z}}{1-\sqrt{z}}\right)\right]^{2}, z \in E
$$

where the symbol $\prec$ denotes subordination. This class was introduced by Ronning [8] and it is denoted by $U C V(\alpha)$. The class of all analytic functions $f(z) \in A$ for which $\frac{z f^{\prime}(z)}{f(z)}$ lies in the parabolic region is denoted by $S_{p}(\alpha)$ and defined as follows. 
Definition C. A function $f(z)$ is said to be in the class $S_{p}(\alpha)$ if for all $z \in E$,

$$
\left|\frac{z f^{\prime}(z)}{f(z)}-1\right| \leq \operatorname{Re}\left\{\frac{z f^{\prime}(z)}{f(z)}\right\}-\alpha, \text { for }-1<\alpha<1 .
$$

This implies $f \in S_{p}(\alpha)$ for $z \in E$ if and only if $\frac{z f^{\prime}(z)}{f(z)}$ lies in the region $\Omega_{\alpha}$ bounded by a parabola with vertex at $\left(\frac{1+\alpha}{2}, 0\right)$ and parameterized by

$$
\frac{t^{2}+1-\alpha^{2}+2 i t(1-\alpha)}{2(1-\alpha)} \text { for any real } t .
$$

It is known [8] that the function

$$
P_{\alpha}(z)=1+\frac{2(1-\alpha)}{\pi^{2}}\left[\log \frac{1+\sqrt{z}}{1-\sqrt{z}}\right]^{2}
$$

maps the unit disk $E$ on to the parabolic region $\Omega_{\alpha}$ (The branch $\sqrt{z}$ is choosen in such a way that $\operatorname{Im} \sqrt{z} \geq 0)$. Then from the above definition $f \in A$ is in the class $S_{p}(\alpha)$ if and only if $\frac{z f^{\prime}(z)}{f(z)} \prec P_{\alpha}(z)$.

The notion of $\delta$-neighbourhood was first introduced by St. Ruscheweyh [11].

Definition D. For $\delta \geq 0$, the $\delta$-neighbourhood of $f(z)=z+\sum_{n=2}^{\infty} a_{n} z^{n} \in A$ is defined by

$$
N_{\delta}(f)=\left\{g(z)=z+\sum_{n=2}^{\infty} b_{n} z^{n}: \sum_{n=2}^{\infty} n\left|a_{n}-b_{n}\right| \leq \delta\right\} .
$$

Recently Padmanabhan [4] has introduced the neighbourhoods of functions in the calss $S_{p}$ and studied various properties.

In this paper we introduce a new class of functions and study the properties of neighbourhoods of functions in this class which generalizes the recent results of Padmanabhan [4] and Ronning [8] Ram Reddy and Thirupathi Reddy [6, 7].

First let us state a lemma which is needed to establish our results in the sequel.

Lemma $\mathbf{A}([10])$. If $\phi$ is a convex univalent function with $\phi(0)=0=\phi^{\prime}(0)-1$ in the unit disc $E$ and $g$ is starlike univalent in $E$, then for each analytic function $F$ in $E$, the image of $E$ under $\frac{\left(\phi * F_{g}\right)(z)}{(\phi * g)(z)}$ is a subset of the convex hull of $F(E)$.

\section{Main results}

Definition 1. Let $S P_{s}(\alpha, \beta)$ be the class of all function $f \in S$ with the property that,

$$
\left|\frac{2 z f^{\prime}(z)}{f(z)-f(-z)}-(\alpha+\beta)\right| \leq \operatorname{Re}\left(\frac{2 z f^{\prime}(z)}{f(z)-f(-z)}+\alpha-\beta\right)
$$

$z \in E, 0<\alpha<\infty$ and $0 \leq \beta<1$. 
This implies that $f \in S P_{S}(\alpha, \beta)$ for $z$ in $E$ which $\frac{2 z f^{\prime}(z)}{f(z)-f(-z)}$ lies in $\Omega=\{\mathrm{w}:|w-(\alpha+\beta)| \leq$ $\operatorname{Re}(w+\alpha-\beta)\}$.

That is that portion of the plane which contains $w=1$ and is bouned by the parabola $y^{2}=4 \alpha(x-\beta)$ whose vertex is the point $w=\beta$. Under the choice of $0<\alpha<\infty, 0 \leq \beta<1$, $\Omega \subset\{w: \operatorname{Re} w>\beta\}$ and hence $S P_{s}(\alpha, \beta) \subset S T(\beta)$ the class of starlike univalent functions of order $\beta$.

Definition 2. Let $U C V_{s}(\alpha, \beta)$ be the class of all function $f(z) \in S$ such that $\frac{2 z f^{\prime}(z)}{f(z)-f(-z)} \in S P_{s}(\alpha, \beta)$.

We now give a characterization of the class $S P_{S}(\alpha, \beta)$ in terms of convolution.

Definition 3. Let $S P_{s}^{\prime}(\alpha, \beta)$ be the class of all function $H(z)$ in $A$ of the form

$$
H(z)=\frac{4 \alpha}{4 \alpha(1-\beta)-4 \alpha i t-t^{2}}\left[\frac{z}{(1-z)^{2}}-\frac{t^{2}+4 \alpha \beta+4 i \alpha t}{4 \alpha}\left(\frac{z}{1-z^{2}}\right)\right]
$$

for $0<\alpha<\infty, 0 \leq \beta<1$ and $t$ is real.

Theorem 1. A function $f$ in $A$ is in $S P_{s}(\alpha, \beta)$ if and only iffor all $z$ in $E$ for all

$$
H(z) \in \operatorname{SP}_{\mathrm{S}}^{\prime}(\alpha, \beta) \text { and } \frac{(f * H)(z)}{z} \neq 0 .
$$

Proof. Let us assume that for $f \in A$ and $\frac{(f * H)(z)}{z} \neq 0$, for all, $H(z) \in S P_{S}^{\prime}(\alpha, \beta)$ and for $z \in E$. From the definition of $H(z)$ it follows that

$$
\frac{(f * H)(z)}{z}=\frac{4 \alpha}{4 \alpha(1-\beta)-4 \alpha i t-t^{2}}\left[z f^{\prime}(z)-\frac{t^{2}+4 \alpha \beta+4 \alpha i t}{4 \alpha}\left(\frac{f(z)-f(-z)}{2}\right)\right] \neq 0,
$$

or equivalently

$$
\frac{2 z f^{\prime}(z)}{f(z)-f(-z)} \neq \frac{t^{2}+4 \alpha \beta+4 \alpha i t}{4 \alpha} \text {, for } t \in R \text {. }
$$

This means that $\frac{2 z f^{\prime}(z)}{f(z)-f(-z)}$ lies completely either inside $\Omega$ or complement of $\Omega$ for all $z$ in E. At $z=0, \frac{2 z f^{\prime}(z)}{f(z)-f(-z)}=1 \in \Omega$. So that $\frac{2 z f^{\prime}(z)}{f(z)-f(-z)} \subset \Omega$, which shows that $f \in \operatorname{SP}_{S}(\alpha, \beta)$.

Conversely let $f \in S P_{s}^{\prime}(\alpha, \beta)$. Hence $\frac{2 z f^{\prime}(z)}{f(z)-f(-z)}$ lies in $\Omega$ which contains $w=1$ and bounded by the parabola $y^{2}=4 \alpha(x-\beta)$. Any point on the parabola can be taken as $\frac{t^{2}+4 \alpha \beta}{4 \alpha}+i t$ for any $t \in R$. So $f \in S P_{S}(\alpha, \beta)$ if and only if $\frac{2 z f^{\prime}(z)}{f(z)-f(-z)} \neq \frac{t^{2}+4 \alpha \beta+4 \alpha i t}{4 \alpha}$ or equivalently

$$
f(z) *\left[\frac{z}{(1-z)^{2}}-\frac{t^{2}+4 \alpha \beta+4 \alpha i t}{4 \alpha}\left(\frac{z}{1-z^{2}}\right)\right] \neq 0 \text {, for } z \in E-\{0\} .
$$

Normalizing the function with in the brackets we get $\frac{(f(z) * H)(z)}{z} \neq 0, z \in E$, where $H(z)$ is the function in Definition 3.

To establish the $T-\delta$ neighbourhoods of functions belonging to the class $S P_{s}(\alpha, \beta)$ we need the following lemmas. 
Lemma 1. Let $H(z)=z+\sum_{k=2}^{\infty} c_{k} z^{k} \in S P_{s}^{\prime}(\alpha, \beta)$ then $\left|c_{k}\right| \leq \frac{k-\beta}{1-\beta}, \forall k \geq 2$.

Proof. Let $H(z)=z+\sum_{k=2}^{\infty} c_{k} z^{k} \in S P_{s}^{\prime}(\alpha, \beta)$, then for any real $t$

$$
\begin{aligned}
H(z) & =\frac{4 \alpha}{4 \alpha(1-\beta)-4 \alpha i t-t^{2}}\left[\frac{z}{(1-z)^{2}}-\frac{t^{2}+4 \alpha \beta+4 \alpha i t}{4 \alpha}\left(\frac{z}{1-z^{2}}\right)\right] \\
& =\frac{4 \alpha}{4 \alpha(1-\beta)-4 \alpha i t-t^{2}}\left[\left(z+2 z^{2}+\cdots\right)-\frac{t^{2}+4 \alpha \beta+4 \alpha i t}{4 \alpha}\left(z+z^{3}+\cdots\right)\right] \\
& =z+\sum_{k=2}^{\infty} c_{k} z^{k}
\end{aligned}
$$

Then comparing the coefficients on either side, we get

$$
c_{k}= \begin{cases}\frac{4 \alpha}{4 \alpha(1-\beta)-4 \alpha i t-t^{2}}, & \text { when } k \text { is even, } \\ \frac{4 \alpha(1-\beta)-t^{2}-4 \alpha i t}{4 \alpha(1-\beta)-t^{2}-4 \alpha i t}, & \text { when } k \text { is odd. }\end{cases}
$$

Hence when $k$ is even, which yields on simplication $\left|c_{k}\right| \leq \frac{k-\beta}{1-\beta}$ and when $k$ is odd we have

$$
\begin{aligned}
\left|c_{k}\right|^{2} & =\frac{\left(4 \alpha(k-\beta)-t^{2}\right)^{2}+16 \alpha^{2} t^{2}}{\left(4 \alpha(1-\beta)-t^{2}\right)^{2}+16 \alpha^{2} t^{2}} \leq \frac{\left(4 \alpha(k-\beta)-t^{2}\right)^{2}+16 \alpha^{2} t^{2}}{\left(4 \alpha(1-\beta)+t^{2}\right)^{2}} \text { if } \alpha \geq 1-\beta \\
& =\frac{16 \alpha^{2}(k-\beta)^{2}-8 \alpha(k-\beta) t^{2}+t^{4}+16 \alpha^{2} t^{2}}{16 \alpha^{2}(1-\beta)^{2}-8 \alpha(1-\beta) t^{2}+t^{4}} \\
& =\frac{16 \alpha^{2}(k+1-2 \beta)^{2}(k-1)-8 \alpha t^{2}(k+1-2 \alpha-2 \beta)}{\left(4 \alpha(1-\beta)+t^{2}\right)^{2}}+1 \\
& =\frac{8 \alpha(k+1-2 \beta-2 \alpha)\left(2 \alpha(k-1)-t^{2}\right)+32 \alpha^{3}(k+1)}{\left(4 \alpha(1-\beta)+t^{2}\right)^{2}}+1 \\
& \leq \frac{16 \alpha^{2}(k-1)(k+1-2(\alpha+\beta))+32 \alpha^{3}(k-1)}{16 \alpha^{2}(1-\beta)^{2}}+1 \\
& =\frac{(k-1)(k+1-2(\alpha+\beta))+(k-1) 2 \alpha}{(1-\beta)^{2}}+1 \\
& =\frac{(k-1)(k+1-2 \beta)}{(1-\beta)^{2}}+1=\frac{(k-\beta)^{2}}{(1-\beta)^{2}}
\end{aligned}
$$

Therefore

$$
\left|c_{k}\right| \leq \frac{k-\beta}{1-\beta}, \quad \forall k \geq 2
$$

Lemma 2. For $f \in A$ and for every $\varepsilon \in C$ such that $|\varepsilon|<\delta$ if $F_{\varepsilon}(z)=\left\{\frac{f(z)+\varepsilon z}{1+\varepsilon}\right\} \in S P_{s}(\alpha, \beta)$, then for every $H(z) \in S P_{s}^{\prime}(\alpha, \beta)$ implies $\frac{(f * H)(z)}{z} \neq \delta, \forall z \in E$. 
Proof. Let $F_{\varepsilon}(z) \in S P_{S}(\alpha, \beta)$, then by Theorem 1

$$
\frac{(f * H)(z)}{z} \neq 0 \quad \forall H(z) \in S P_{s}^{\prime}(\alpha, \beta)
$$

and $z \in E$. Equivalently

$$
\frac{(f * H)(z)+\varepsilon z}{(1+\varepsilon) z} \neq 0 \quad \text { or } \quad \frac{(f * H)(z)}{z} \neq-\varepsilon .
$$

Hence

$$
\left|\frac{(f * H)(z)}{z}\right| \geq \delta, \quad \forall z \in E .
$$

Theorem 2. For $f \in A$ and for every $\varepsilon \in C$ and for $|\varepsilon|<\delta<1$, assume $F_{\varepsilon}(z) \in S P_{s}(\alpha, \beta)$. If further $\alpha \geq 1-\beta$ then $T N_{\delta}(f) \subset S P_{s}(\alpha, \beta)$ with $\left\{T_{k}\right\}=\left\{\frac{k-\beta}{1-\beta}\right\}$.

Proof. Let $T_{k}=\frac{k-\beta}{1-\beta}$ and $g(z)=z+\sum_{k=2}^{\infty} b_{k} z^{k}$. Then for $\alpha \geq 1-\beta$, from Lemma 1 we have,

$$
\begin{aligned}
\left|\frac{1}{z}(g * H)(z)\right| & =\left|\frac{1}{z}((g * f) H)(z)+\frac{1}{z}(f * H)(z)\right| \\
& \geq\left|\frac{(f * H)(z)}{z}\right|-\left|\frac{((g-f)(z) * H)(z)}{z}\right| \\
& >\left|\frac{(f * H)(z)}{z}\right|-\sum_{k=2}^{\infty}\left|b_{k}-a_{k}\right|\left(\frac{k-\beta}{1-\beta}\right) .
\end{aligned}
$$

Since

$$
F_{\varepsilon}(z)=\frac{f(z)+\varepsilon z}{1+\varepsilon} \in S P_{s}(\alpha, \beta), \quad F_{\varepsilon}(z) * H(z) \neq 0, \quad \forall H(z) \in S P_{s}^{\prime}(\alpha, \beta), z \in E
$$

or

$$
\frac{(f * H)(z)}{z} \neq-\varepsilon
$$

which is equivalently

$$
\left|\frac{(f * H)(z)}{z}\right|>\delta \text { for }|\varepsilon|<\delta
$$

Therefore $g \in T N_{\delta}(f)$ we get from (12) that $\left|\frac{(g * H)(z)}{z}\right|>0$ and hence $\frac{(g * H)(z)}{z} \neq 0$ in $E \forall H(z) \in S P_{s}^{\prime}(\alpha, \beta)$ and $\alpha \geq(1-\beta)$ there by showing $g \in S P_{s}(\alpha, \beta)$. This proves that $T N_{\delta}(f) \subset S P_{s}(\alpha, \beta)$.

Next we will show that the class $S P_{s}(\alpha, \beta)$ is closed under convolution with functions $f$ which are convex univalent in $E$.

Lemma 3. If $g \in S P_{s}(\alpha, \beta)$ then $G(z) \in S P_{s}(\alpha, \beta) \subset S T$ where $G(z)=\frac{g(z)-g(-z)}{2}$. 
Proof. Since $g \in S P_{S}(\alpha, \beta), \frac{2 z g^{\prime}(z)}{g(z)-g(-z)} \in \Omega$. Now

$$
\frac{z G^{\prime}(z)}{G(z)}=\frac{z g^{\prime}(z)}{2 G(z)}+\frac{(-z) g^{\prime}(-z)}{2 G(-z)}=\frac{\zeta_{1}}{2}+\frac{\zeta_{2}}{2}=\zeta_{3} \text { where } \zeta_{1} \text { and } \zeta_{2} \in \Omega .
$$

Since $\Omega$ is convex $\zeta_{3} \in \Omega$ and hence $\frac{z G^{\prime}(z)}{G(z)} \in \Omega$. It can be easily seen that $S P_{s}(\alpha, \beta) \subset S T$. Thus $G(z) \in S P_{s}(\alpha, \beta) \subset S T$.

Theorem 4. Let $f(z) \in C V$ the class of convex functions and $g(z) \in S P_{s}(\alpha, \beta)$. Then $(f * g)(z) \in$ $S P_{s}(\alpha, \beta)$.

Proof. Let $f(z) \in C V$ and $g(z) \in S P_{s}(\alpha, \beta), G(z)=(g(z)-g(-z)) / 2$ and $\Omega$ is a convex domain. Since $g(z) \in S P_{S}(\alpha, \beta), G(z) \in S T$ by Lemma 3. Hence by an application of Lemma A we get

$$
\frac{z(f * g)^{\prime}(z)}{(f * G)(z)}=\frac{\left(f * z g^{\prime}\right)(z)}{(f * G)(z)}=\frac{f * \frac{z g^{\prime}(z)}{G(z)} G(z)}{(f * G)(z)} \subset \overline{C_{0}}\left(\frac{z g^{\prime}(z)}{G(z)}\right) \subset \Omega .
$$

Since $\Omega$ is convex and $g \in S P_{s}(\alpha, \beta)$. This proves that $(f * g)(z) \in S P_{s}(\alpha, \beta)$.

Theorem 5. If $f \in U C V_{s}(\alpha, \beta)$, then $\frac{f(z)+\varepsilon z}{1+\varepsilon} \in S P_{s}(\alpha, \beta)$ for $|\varepsilon|<\frac{1}{4}$.

Proof. Let $f(\mathrm{z})=z+\sum_{k=2}^{\infty} a_{k} z^{k}$. Then

$$
\begin{aligned}
\frac{f(z)+\varepsilon z}{1+\varepsilon} & =\frac{z(1+\varepsilon)+\sum_{k=2}^{\infty} a_{k} z^{k}}{1+\varepsilon}=\frac{f(z) *\left[z(1+\varepsilon)+\sum_{k=2}^{\infty} z^{k}\right]}{1+\varepsilon} \\
& =f(z) * \frac{\left(z-\frac{\varepsilon}{1+\varepsilon} z^{2}\right)}{(1-z)}=f(z) * h(z)
\end{aligned}
$$

where $h(z)=\frac{\left[z-\frac{\varepsilon}{1+\varepsilon} z^{2}\right]}{(1-z)}$

$$
\frac{z h^{\prime}(z)}{h(z)}=\frac{\left[z-\frac{2 \varepsilon}{1+\varepsilon} z^{2}\right]}{\left[z-\frac{\varepsilon}{1+\varepsilon} z^{2}\right]}+\frac{z}{1-z}=\frac{-\rho z}{1-\rho z}+\frac{1}{1-z}, \quad \text { where } \quad \rho=\frac{\varepsilon}{1+\varepsilon} .
$$

Hence $|\rho|<\frac{\varepsilon}{1-|\varepsilon|}<1 / 3$ gives $|\varepsilon|<1 / 4$. Thus

$$
\operatorname{Re}\left\{\frac{z h^{\prime}(z)}{h(z)}\right\} \geq \frac{1-2|\rho||z|-|\rho||z|^{2}}{(1-|\rho||z|)(1+|z|)}>0
$$

if $|\rho|\left(|z|^{2}+2|z|\right)-1<0$, which is true only when $|\rho|<1 / 3$. Therefore $h$ is starlike in $E$ for $|\varepsilon|<1 / 4$. Now $h(z) * \log \left(\frac{1}{1-z}\right)=\int_{0}^{z} \frac{h(t)}{t} d t$ is a convex function and

$$
(f * h)(z)=(h * f)(z)=\left[h(z) * z f^{\prime}(z) * \log \left(\frac{1}{1-z}\right)\right]=z f^{\prime}(z) *\left[h(z) * \log \left(\frac{1}{1-z}\right)\right] .
$$


Hence $f(z) \in U C V_{s}(\alpha, \beta)$ implies $z f^{\prime}(z) \in S P_{s}(\alpha, \beta)$ and $h(z) * \log \left(\frac{1}{1-z}\right) \in C V$.

Now by Theorem 4 we have

$$
z f^{\prime}(z) *\left[h(z) * \log \left(\frac{1}{1-z}\right)\right] \text { is in } S P_{s}(\alpha, \beta) .
$$

Therefore

$$
(f * h)(z)=\frac{f(z)+\varepsilon z}{1+\varepsilon} \in S P_{s}(\alpha, \beta) \text { for } \quad|\varepsilon|<\frac{1}{4} .
$$

Remark 1. By letting $\varepsilon=0$ in Theorem 5 we get the following result.

Corollary 6. If $f \in U C V_{s}(\alpha, \beta)$ then $f(z) \in S P_{s}(\alpha, \beta)$.

Theorem 7. Let $f \in U C V_{s}(\alpha, \beta)$ and $\alpha \geq 1-\beta$. Then $T N_{1 / 4}(f) \subset S P_{s}(\alpha, \beta)$.

Proof. Let $f \in U C V_{s}(\alpha, \beta)$. Then from Theorem 5 we have $\frac{f(z)+\varepsilon z}{1+\varepsilon} \in S P_{s}(\alpha, \beta)$ for $|\varepsilon|<\frac{1}{4}$. Then an application of Theorem 2 gives when $\alpha \geq 1-\beta, T N_{1 / 4}(f) \subset S P_{S}(\alpha, \beta)$.

\section{Acknowledgements}

The authors would like to thank the referee of the paper for his helpful comments.

\section{References}

[1] A. W. Goodman, On uniformly convex functions, Ann. Polon. Math., 56(1991), 87-92.

[2] A. W. Goodman, On uniformly starlike functions, J. Math. Anal. Appl., 155(1991), 364-370.

[3] W. Ma and D. Minda, Uniformly convex functions, Ann. Polon, Math., 57 (1992), 165-175.

[4] K. S. Padmanabhan, On uniformly convex functions in the unit disk, J. Analysis, 2 (1994), 87-96.

[5] R. Parvatham and M. Premabai, Neighbourhoods of uniformly starlike functions with respect to symmetric points, Bull. Cal. Math. Soc., 88(1996), 155-160.

[6] T. Ram Reddy and P. Thirupathi Reddy, Neighbourhoods of a certain subclass of SP( $\beta$ ), J. Analysis, 15 (2007), 239-245.

[7] T. Ram Reddy and P. Thirupathi Reddy, Neighbourhoods of a certain subclass of SP $(\alpha)$ with respect to symmetric points, Bull. Cal. Math. Soc., 101(2009), 359-366.

[8] F. Ronning, On starlike functions associated with parabolic regions, Ann. Univ. Mariae Curie-Sklodowska. Sect. A, 45 (1991), 117-122.

[9] F. Ronning, Uniformly convex functions and a corresponding class of starlike functions, Proc. Amer. Math. Soc. 118(1993), 189-196.

[10] St. Ruscheweyh and T. Sheil-Small, Hadamard products of schlicht functions and the Polya-Schoenberg Conjecture, Comment. Math. Helv., 48 (1973), 119-135.

[11] St. Ruscheweyh, Neighbourhoods of univalent functions, Proc. Amer. Math. Soc., 81 (1981), 521-527.

Department of Mathematics, Kakatiya University, Warangal 506009, A.P., India.

E-mail: reddypt2@yahoo.com 